\title{
Regenerative Medicine to Reduce the Side Effects from Radiotherapy Causing Skin Cancer, Fibrosis, Neuropathic Pain and Hair Loss
}

\author{
E. Russell Vickers ${ }^{1}{ }^{*}$, Junqing Liang ${ }^{2}$, Peter G. Vickers ${ }^{1}$, Haitao Wen ${ }^{1}$ \\ ${ }^{1}$ Clinical Stem Cells PL, Sydney, Australia \\ ${ }^{2}$ Inner Mongolia Tumor Hospital, Hohhot, China \\ Email: ^manager@clinicalstemcells.com
}

How to cite this paper: Vickers, E.R., Liang, J.Q., Vickers, P.G. and Wen, H.T. (2021) Regenerative Medicine to Reduce the Side Effects from Radiotherapy Causing Skin Cancer, Fibrosis, Neuropathic Pain and Hair Loss. Journal of Cancer Therapy, 12, 461-477.

https://doi.org/10.4236/jct.2021.128040

Received: July 20, 2021

Accepted: August 17, 2021

Published: August 20, 2021

Copyright $\odot 2021$ by author(s) and Scientific Research Publishing Inc. This work is licensed under the Creative Commons Attribution International License (CC BY 4.0).

http://creativecommons.org/licenses/by/4.0/

(c) $\underset{\mathrm{By}}{\text { Oi }}$ Open Access

\begin{abstract}
Surgery, chemotherapy and radiotherapy are the gold standard treatments used for cancer. Side effects from medical radiation can be significant, particularly external beam therapy to the breast, and head and neck regions causing fibrosis, secondary skin cancer, hair loss, oral mucositis and neuropathic pain. There is significant psychological stress, depression, anxiety and loss of self esteem particularly for female patients. Similar types of space radiation are known to damage the health of astronauts. Current treatments for scarring, tissue loss, hair loss and neuropathic pain are a high priority but inadequate. Regenerative medicine is a new and comprehensive approach to potentially treat complex medical conditions from radiation damage. Regenerative medicine combines a systematic evidence based approach with the use of herbal medicine, stem cells, peptides and 3D tissue engineering. These fields use sophisticated technology to identify the respective molecular mechanisms of upregulation and protection of healthy cells and down regulation of cancer cells. The regenerative medicine strategies of stem cells and plant polyphenols suggest there is significant potential for rapid clinical translation to alleviate the side effects associated with radiotherapy.
\end{abstract}

\section{Keywords}

Herbal Medicine, Polyphenols, Radiation, Radiotherapy, Regenerative Medicine, Stem Cells

\section{Introduction}

Cancer is a major cause of death worldwide with an estimated 19.3 million new cancer cases and almost 10.0 million cancer deaths occurring in 2020. Female 
breast cancer has surpassed lung cancer as the most commonly diagnosed cancer, with an estimated 2.3 million new cases (11.7\%) [1]. Research into cancer survival has evolved considerably with the US National Cancer Institute defining survivorship as the focus on "the health and life of a person with cancer post treatment until the end of life." [2] It reflects the paradigm shift of human health concern to cover the attributes of the physical and biopsychosocial domains. In addition, attention is attached to the personal and governmental socioeconomic issues of cancer. The $21^{\text {st }}$ century approach to cancer includes its diagnosis, treatment, side effects of treatment in the short term and long term, and the overall quality of life.

Radiotherapy to treat cancer can be performed as a single modality technique for inoperable brain cancer and to reduce pain and inflammation from metastatic spinal cord lesions. The majority of cancer treatments utilize surgery to excise the lesion completely, or to debulk the tumor, followed by radiotherapy as the acceptable gold standard approach. The selection of the single versus multi-modal therapy is decided by an oncology team and the treatment decision made on an individual basis considering the variables of tumor location, grade, cell type, local or regional spread, metastatic potential or presence, and adjacent anatomical structures. Radiation has a distinct advantage compared with surgery due to its minor or non-invasive approach in medically compromised patients. In addition, radiotherapy is applicable where the primary tumor or secondaries are located in high risk surgical sites of the brain, brain stem and spinal cord. The oncology team will carefully consider the patient's general health, co-existing medical conditions, psychological parameters and family support.

There has been good progress in the delivery methods of radiation to reduce its damaging effects to adjacent healthy tissue. However, two important anatomical areas are subject to the effects of field radiation that can cause significant side effects. The breast, and oral cavity and facial region are areas of high priority for the patient. These areas are highly personal with enormous psychological and social consequences for the patient if they are irreparably damaged during treatment. An example would be the postsurgical loss of an intimate personal part such as the breast that is replaced by significant scars (Figure 1). External beam radiotherapy can produce persistent painful scars and long term alopecia (hair loss).

Cancer treatment to the breast and orofacial regions frequently uses a combination of ablative surgery and radiotherapy. Post-treatment breast complications of surgery and radiotherapy can be unsightly scars of a key body part and the onset of neuropathic pain. For women this can have major negative psychological implications triggering anxiety, depression, stress, and loss of personal esteem (Figure 2). The breast and orofacial region have a dense neurosensory network that is necessary for functioning. Unfortunately nerve trauma in the form of neurotmesis from scalpel incisions induces neuroma formation causing spontaneous pain (Figure 3). High energy radiation damage to neurons results in the loss of the protective myelin sheaths producing sharp, shooting and burning 

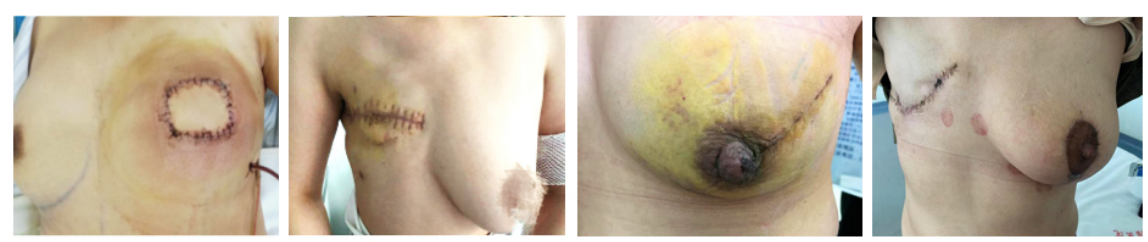

Figure 1. Patient examples of significant scarring of breast following mastectomy in four women.

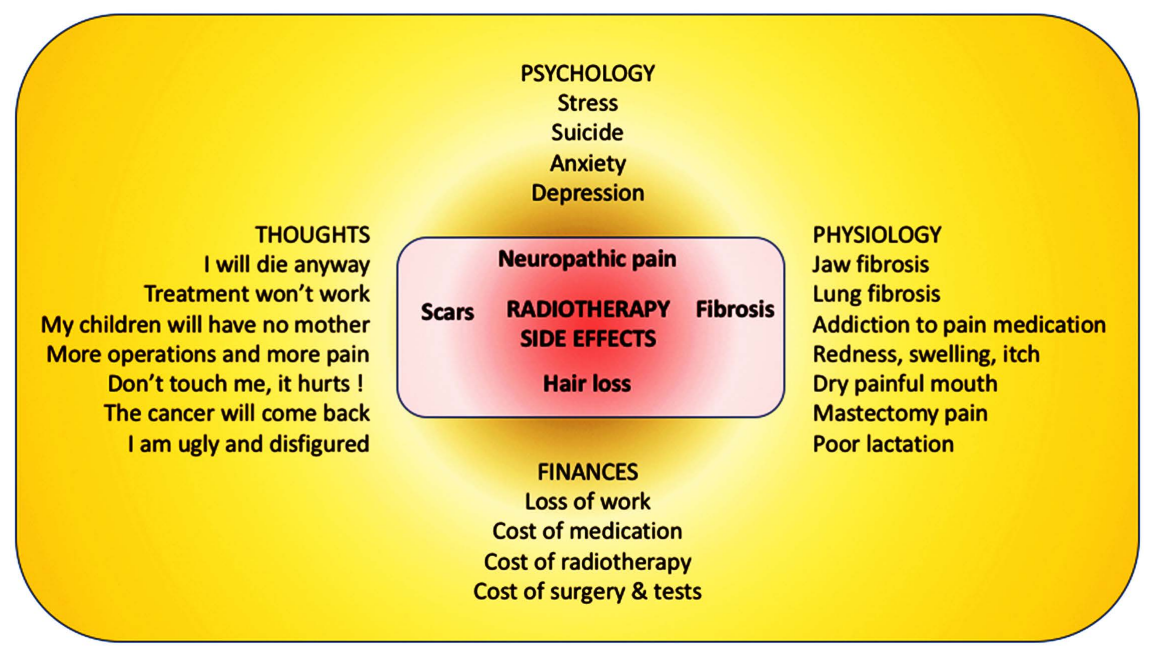

Figure 2. Common side effects of radiotherapy to the head and breast regions causing scars, fibrosis, neuropathic pain and hair loss. A biopsychosocial analysis of the scope of negative conditions as a result of these complications including negative thoughts, financial loss, psychological stress and physical deterioration.

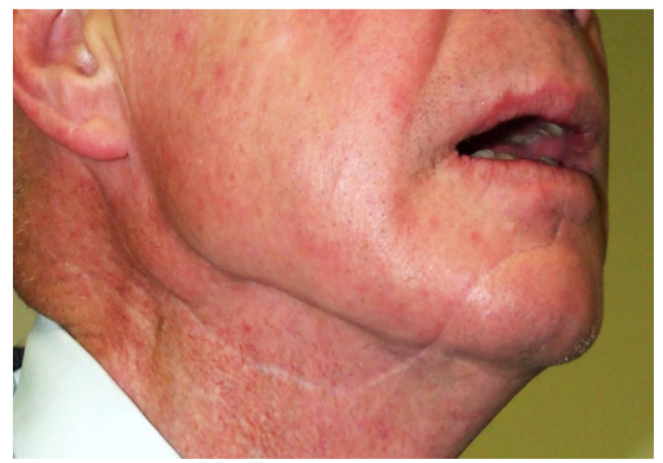

Figure 3. A 65 year old Caucasian male with neuropathic pain in the healed incision line of the lip. The incision was performed for surgical access to a squamous cell carcinoma situated on the hard palate. The pain intensity was reported at $6 / 10$ and pain medication of codeine, amitriptyline and carbamazepine was ineffective. Topical capsaicin was applied for several weeks and abolished the pain.

neuropathic pain qualities. The purpose of breast radiotherapy is obliteration of residual cancer cells adjacent to the surgical site with a three stage approach of $\mathrm{CT}$ simulation, contouring and planning. Immediate post radiation complications of edema, necrosis and pneumonia are quickly observed. The immediate to late stage problems are seen with breast and pulmonary fibrosis, cardiomyopa- 
thy and radiation-induced cancer [3]. There is significant prevalence of radiation fibrosis 12 months after treatment and was shown to occur in $90.5 \%$ of patients with $17.1 \%$ being symptomatic [4].

Where the patient has oropharyngeal (head and neck) cancer the surgical plan is to attempt an excisional approach with generous surgical margins of the primary tumor and regional head and neck lymph nodes. Alternately, if the tumor is large and close to critical nerves or vessels then the approach is to debulk the tumor for short term improvement to maintain facial esthetics, and jaw and nasal function. The head and neck region, arguably, is the most anatomically complex part of the body with multiple cranial nerves, carotid arterial supply and its branches, venous complexity, lymphatic drainage, salivary glands and jaw muscles. The face, teeth, tongue and jaws have vital functions of communication and eating to obtain adequate nutritional requirements. Substantial facial tissue loss can be improved for function and esthetics by vascular flap transfers and prosthetics. However, surgical intervention can result in extensive facial scars. Major residual scars can be unsightly and cause psychological distress. Radiation induced skin reactions are frequently observed. It has been reported that $>70 \%$ of patients with malignant tumors need radiotherapy and 95\% develop skin redness, irritation and ulceration due to oxidative stress [5]. As early as 1970 it was shown that localized facial skin cancer is an unfortunate consequence of radiotherapy that has targeted oral cancers (Figure 4, Figure 5). The investigators reported that most tumors were of basal cell carcinoma diagnosis and developed
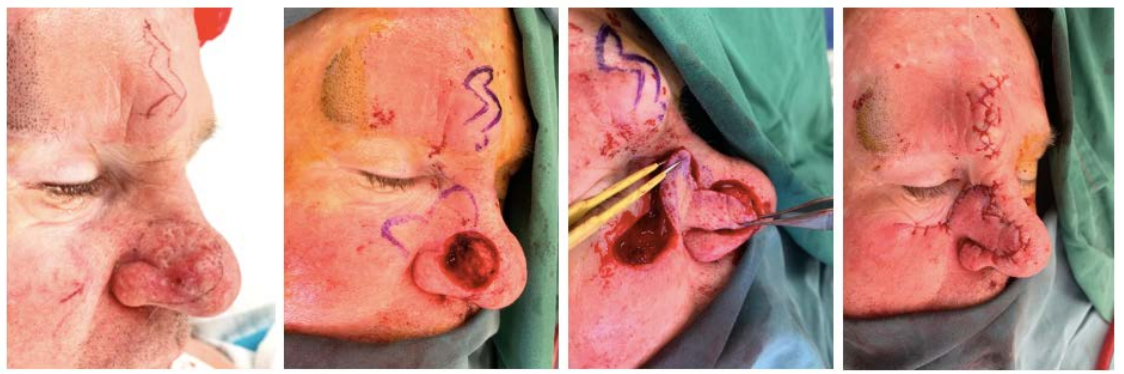

Figure 4. Development of a basal cell carcinoma in a 58 year old male. The lesion was situated on the nose and occurred 15 years after radiation treatment following excision of an oral squamous cell carcinoma.
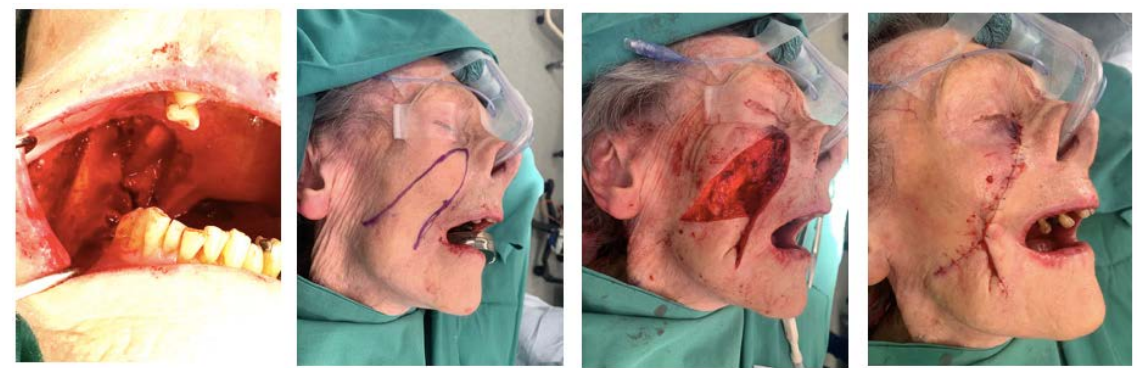

Figure 5. An 80 year old female undergoing excision of recurrence of a squamous cell carcinoma that occurred 20 years previously in the retromolar trigone region. Post-surgical radiation treatment was performed after the original surgery. 
quickly in the younger patients. Centrally located lesions occurred frequently in the nose and circumoral regions [6]. The onset of rapid tooth decay and periodontal disease from radiotherapy magnifies the problem as there is a marked decline in a socially acceptable appearance coupled with a marked decline in tooth function to masticate food for nutritional requirements (Figure 6).

Alopecia is an extremely visible and upsetting condition following radiotherapy and chemotherapy. Despite patients being forewarned about hair loss and options to treat it there remains high level psychological distress in females and children. An excellent review on radiation induced alopecia has recently been reported [7]. Key findings in this report showed persistent or permanent alopecia after cancer was reported in $14 \%$ of childhood cancer survivors and in $30 \%$ of adult breast cancer survivors. Persistent or permanent hair disorders was identified in $60 \%$ of patients from head and neck radiotherapy. The use of wigs and scarves to mask hair loss are used but depression and anxiety is still very common. While hair may grow back there can still be patches of baldness. The quality of regrown hair is less as it is thinner and curlier.

Following radiation treatment to the head and neck the oral mucosa frequently develops radiation mucositis. This is characterized by widespread ulceration of the oral mucosa causing extreme pain on eating and talking with great suffering. The lack of eating can lead to a nutritional deficiency and lower the general health of the recovering patient. Radiotherapy also causes persistent neuropathic pain in the orofacial and breast region. The radiation is directed at tissues that are dense in myelinated A-delta and unmyelinated C sensory nerve fibers. Trigeminal neuropathy is a disabling pain with sharp, shooting, burning and aching qualities [8]. Touching the site increases the pain intensity and is termed mechanical hyperalgesia. The face is very painful to touch such as applying lipstick. For thoracic neuropathy the wearing of clothes increases pain. Invariably the

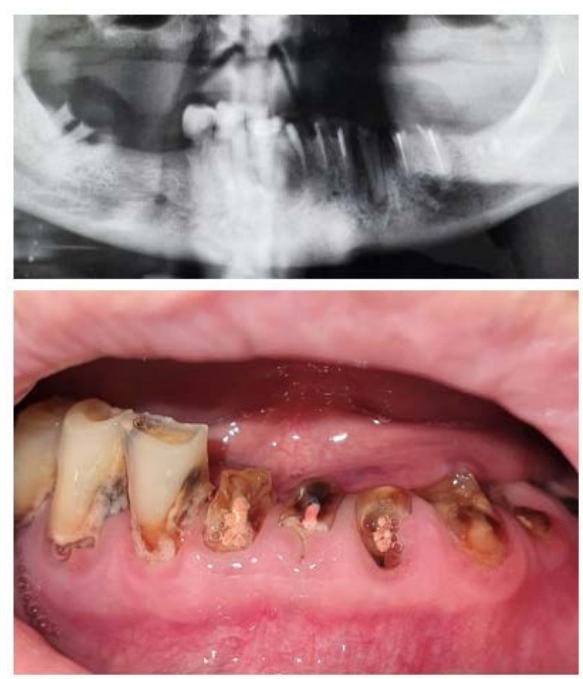

Figure 6. 73 year old male with a history of Hodgkin's lymphoma, squamous cell carcinoma and adenocarcinoma managed with surgery, chemotherapy and radiotherapy. Extensive tooth decay observed on the orthopantogram radiograph and clinical photo. 
afflicted patient retracts from his/her partner avoiding kissing, touching and sex causing relationship problems. Post-mastectomy pain syndrome occurs after surgery in $25 \%-60 \%$ of patients causing neuropathic pain and has a major negative effect on the patient with loss of a woman's personal anatomical part compounded by pain and scars [9]. Persistent neuropathic pain leads to brain neuroinflammation and changes to behavior with depression, anxiety, stress, frustration and anger [10] [11]. At the molecular level the neuropathy has an overexpression of the pain producing peptides such as substance $\mathrm{P}$.

Regenerative medicine is a new and exciting field of medicine that has applications to tissues after radiation. It is a collection of evidence based knowledge involving the core modalities of stem cells, bioscaffolds, exosomes, peptides and natural occurring polyphenols. Most of the published information is from in vitro and ex vivo research, and preliminary clinical trials. Each modality on its own has the capacity to lead to improved outcomes and when combined have the potential to be enormously helpful for patients. There is accumulating published data on each of the modalities but there is a paucity of information applying these fields for radiotherapy patients. The purpose of this article is to outline integrated regenerative medicine approaches from a narrative approach for clinical translation that is applicable to medical radiation complications. In particular, the focus is on the modalities of stem cell methods and natural compounds that could have rapid translation into clinical trials (Figure 7).

\section{Regenerative Medicine Methods}

\subsection{Stem Cells}

There have been enormous leaps in our understanding of stem cells and their

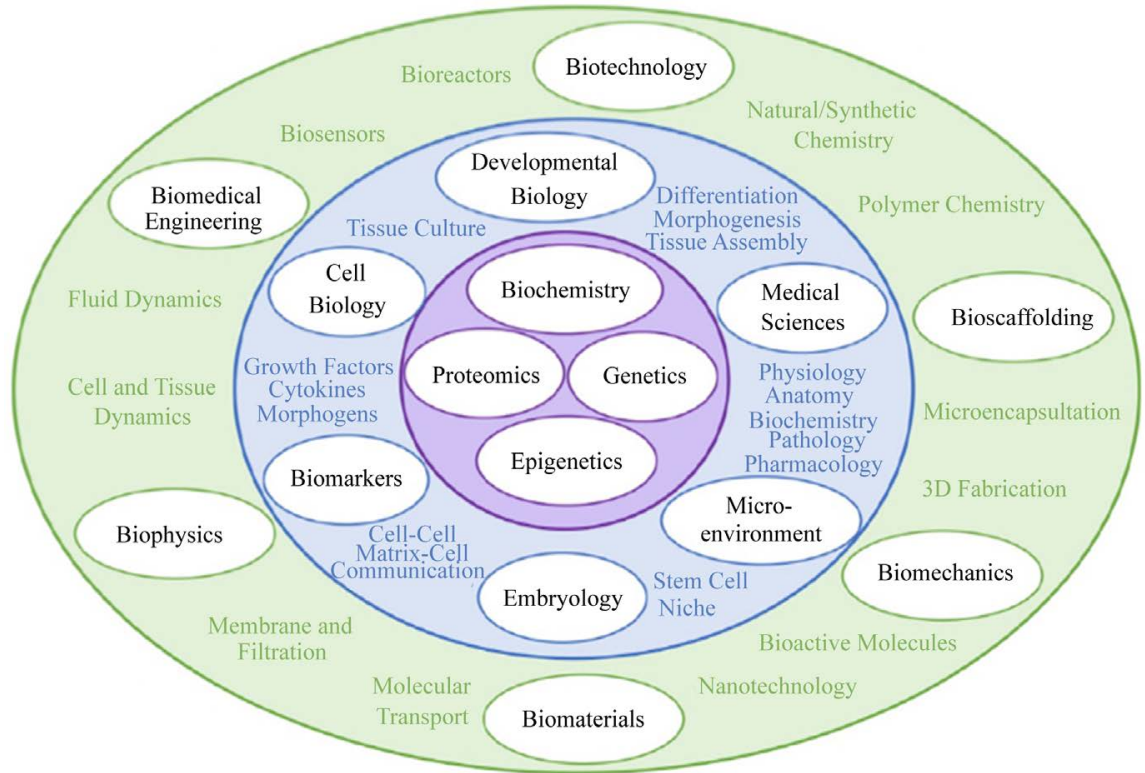

Figure 7. The scope of the integrative fields in regenerative medicine. (c) Shparberg, Braunsteiner \& Vickers (2018) Journal of Stem Cell Research \& Therapy. 
role in regenerative medicine. The four different type of stem cells are umbilical cord, embryonic, induced pluripotent and mesenchymal cells derived from adipose tissue or hematopoietic tissue. All types have undergone clinical trials with adult mesenchymal cells (MSCs) routinely used in conditions such as osteoarthritis with a high margin of safety. Research has indicated that stem cells perform several roles after tissue injury including the release of anti-inflammatory mediators. The second role is for the proliferation and differentiation of cells to form new tissue.

A review on the effect of MSCs after lung irradiation showed the cells migrated to the site of injury and differentiated into specific lung cell types and improved vascularity by forming endothelial cells. The mechanisms occurred via by superoxide dismutase- 1 expression and anti-fibrotic factors hepatocyte growth factor and prostaglandin E2 [12]. From a clinical translation perspective the intravenous administration of MSCs is a simple method to deposit cells into the lungs.

Scarring occurs throughout the body with more obvious dermal scars such as hypertrophic scars (keloids) observed at times. Interest in the stem cell approach is derived from the knowledge that injuries heal without scarring in the first two trimesters when embryonic and mesenchymal stem cells are abundant in the fetus. In addition, the stem cell expression profile (secretome containing peptides) with paracrine activity displays this ability. The ability of non-scarring is lost in the third trimester with a research focus on the scar forming Engrailed-1 gene involved in burn related scars. Peptide compounds reducing scarring have been identified as human beta defensin-2 and -3 , vascular endothelial growth factor, hepatocyte growth factor, and platelet-derived growth factor [13].

Organoids are differentiated stem cell clusters with attributes of the target organ. Lung, cardiac, pancreatic and hepatic organoids have been developed. Importantly, adipose breast cell organoids have been developed to study breast cancer and recently for the potential of 3D bioprinting to regenerate healthy breast tissue [14]. Autologous adipose stem cells have also shown evidence in humans to reduce orofacial neuropathic pain with safety and preliminary efficacy [15]. Stem cells for hair loss particularly for androgenetic alopecia have been in development for several years [16]. Thymulin peptide attached to zinc has been found to be safe and effective in humans for stimulating the anagen phase of hair growth and could possibly increase the rate of hair growth after radiotherapy [17].

\subsection{Natural Compounds}

A recent animal (rat/mouse) model and cellular review of polyphenols to protect brain tissue from gamma radiation has recently been published [18]. It emphasizes that natural compounds when combined with stem cells have a positive effect on cell proliferation and differentiation. Moreover, the plant based polyphenolics have an inhibiting effect on cancer stem cells [19], and breast cancer cells [20]. There are an estimated 20,000 polyphenolic compounds from plants. 
Plants have been on the earth for 290 million years (Ginkgo biloba) and have developed multiple bioactive principles with antioxidant effects found in green vegetables and antiradiation properties in the herb Rhodiola rosea. This article will focus on several well researched polyphenols that could be translated for clinical research to ameliorate radiation side effects (see Figure 8 for chemical structures of compounds).

\subsubsection{Epigallocatechin Gallate}

Epigallocatechin gallate (EGCG) is the major polyphenol found in green tea. Extensive research has been conducted in China on this extremely valuable compound and it has a well established safety profile [21]. When combined with the other green tea bioactives EGCG decreased lung carcinoma by $58 \%$ (mice model) in an aerosol delivery [22]. The compound can enhance cancer cell sensitivity to radiation by regulating apoptosis in the miR-34a/Sirt1/p53 signaling pathway [23]. EGCG has been shown to confer protection to dermal skin cells from ionizing radiation by several mechanisms involving overexpression of heme oxygenase-1 and reduced DNA strand breaks [24]. EGCG promotes neural progenitor cell proliferation and sonic hedgehog pathway activation during adult hippocampal neurogenesis [25]. EGCG has been shown to be effective to reduce scarring of human skin compared with placebo [26]. It may reduce neuropathic pain by reducing ROS [27]. In areas of radiation damage to the skin where secondary bacterial infection can occur the EGCG polyphenol has remarkable antimicrobial activity against pathogenic skin bacteria [28].

\subsubsection{Quercetin}

Quercetin is found in many fruits and vegetables and has high concentrations in kale, broccoli, berries and tomatoes. The compound has many publications for several therapeutic roles including radiation protection on neuronal tissue by reducing oxidative stress in the rat brain exposed to $20 \mathrm{~Gy}$ [29]. Its role as an anti-breast cancer agent has been reported [30]. It has other anticancer tissue properties by cell cycle arrest and apoptosis [31]. It can reduce liver [32] and

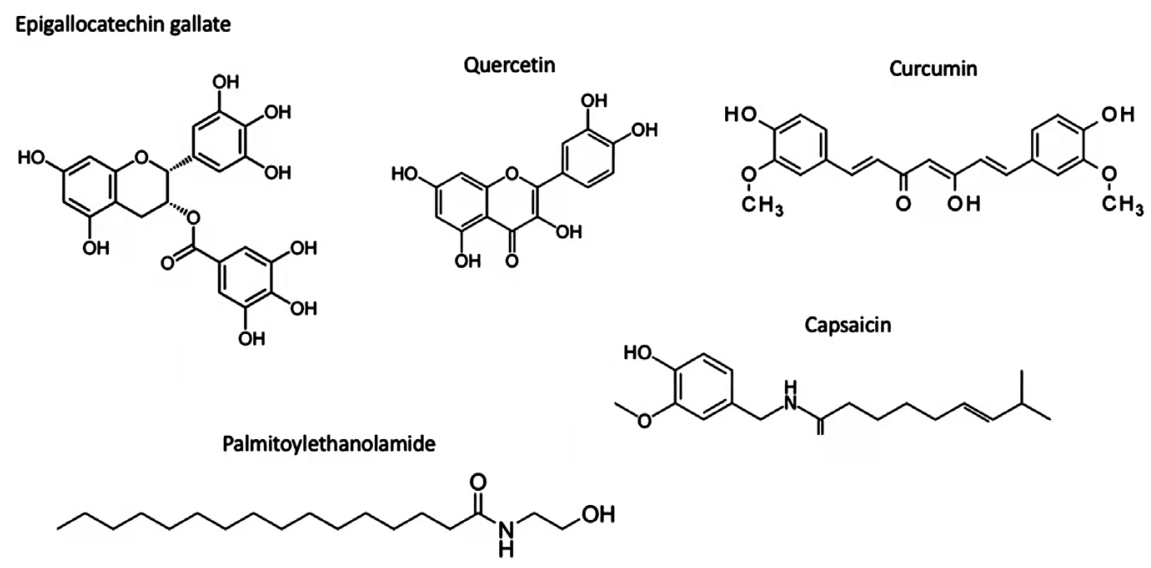

Figure 8. Chemical structures of the natural compounds for regenerative medicine approaches to reduce side effects from medical radiation therapy. 
pulmonary fibrosis [33] and has the potential to decrease dermal scars by altering integrin expression in the extracellular matrix [34]. It promotes human epidermal stem cell proliferation [35]. Quercetin reduces pulmonary fibrosis by reversal of TGF beta $1[36]$.

\subsubsection{Curcumin}

Curcumin is the major bioactive from the turmeric root and is a staple part of the Indian subcontinent diet. A randomized controlled trial has shown that a daily dose of $6 \mathrm{gm}$ of curcumin showed a significant reduction in radiation dermatitis and pain quality, but no effect on erythema. The radiation dose ranged from 42.6 to $50.4 \mathrm{~Gy}$ and the total number of sessions ranged from 16 to 33 [37]. It has also been shown to be protective against radiation induced oxidative stress (rat model) [38]. The compound has been traditionally used in Asian medicine as an antifibrotic and has been supported by recent studies to reduce lung fibrosis [39]. The benefit of curcumin as a novel and safe anticancer agent has received attention with considerable research now published and an excellent recent systematic review showing it has multiple therapeutic effects of inhibiting carcinogenesis, angiogenesis, and tumor growth [40]. It has shown potential for reducing neuropathic pain [41]. Curcumin has demonstrated good regenerative potential for healthy stem cell proliferation and reduces cytotoxicity of stem cells in an unfavorable environment [42].

\subsubsection{Palmitoylethanolamide}

Palmitoylethanolamide (PEA) is a natural endocannabinoid found in humans and mammals. It reduces pain and inflammation as an analgesic and has roles in pain suppression, the immune system, appetite and mood. Recent evidence (rat model) has shown co-administration of PEA with radiotherapy significantly reduced radiation injury and collagen deposition [43]. It also has demonstrated antiproliferative effects (in vitro) against the human adenocarcinoma Caco- 2 cell line and has been suggested as an adjunct to chemotherapy [44]. A recent review of PEA has shown antidepressant effects that were improved with co-administration of citalopram [45]. PEA is widely considered a very safe compound with many countries classifying it as a food. Pharmaceutical grade PEA is obtained from egg yolk, peanut oil and soya bean. It has very few side effects and drug interactions and is available as an orally ingested powder (capsule) form and as a topical cream. It is one of the few compounds to reduce noradrenaline release that is involved in sympathetically maintained pain (a secondary pain to neuropathic pain). The compound can reduce liver fibrosis [46] and tumor cell proliferation [47].

\subsubsection{Capsaicin}

Capsaicin is derived from chillies and capsicum and is structurally similar to the endocannabinoids. It has been traditionally used to reduce cholesterol and lipids. It can be prescribed as a topical formulation to treat oral mucosal and dermal neuropathic pain [48]. A recent comprehensive review found that most stu- 
dies identified capsaicin to have anti-tumor properties for breast cancer, lung cancer, gastric cancer, liver cancer, prostate cancer and bladder cancer [49]. Specific biochemical actions showed it to be an antioxidant by inhibiting lipid peroxidation and protein oxidation, and as a radioprotector by preventing radiation induced loss of the endogenous antioxidant glutathione [50]. Several studies have shown the compound to reduce liver fibrosis [51] but not affect the rate of normal healing in skin (rat model) [52]. Capsaicin has been shown to increase stem cell proliferation but prevent cell fate adipogenic differentiation from bone marrow stem cells [53].

\section{Discussion}

The two fields of stem cells and polyphenols derived from herbal medicines demonstrate the potential of combining these natural techniques to improve radiation treatment outcomes. The loss of a woman's breasts, replaced by painful, ugly scars creates enormous psychological distress. Immediate surgical reconstruction of breast tissue after mastectomy is available for only a limited number of patients. Healthy breast cell organoids to reconstruct lost tissue would have enormous benefits. Regenerative medicine has the possibly of reducing radiation damage to the brain including memory loss. Neurodegenerative effects from brain irradiation show cell apoptosis, demyelination and impaired neurogenesis [54]. Certain polyphenols can stimulate proliferation of the hippocampal neuroprogenitor cells.

In the case of the orofacial region the medical significance of reducing localized complications after radiotherapy directed at the orofacial region should not be overlooked in considering the total welfare of the patient. A pleasing esthetic appearance of the face and teeth underpin self confidence. Radiotherapy causing extensive tooth decay is just one problem when it has been applied after head and neck surgery. When a person has decayed anterior teeth there is a lack of self confidence, reduced employment opportunities and a decline in social interaction. Radiation affected salivary glands reduce salivary output and lowers its buffering capacity leading to rampant tooth decay. Moreover, the teeth can no longer masticate food into smaller pieces so the nutritional uptake in the gastrointestinal tract is reduced. The regional masseter and pterygoid jaw closing muscles can become fibrosed and aggravate chewing inefficiency. The development of fibrosis leads to possible nerve entrapment and spontaneous pain from innocuous stimuli such as touch, thus compounding the sensory aspects of the face. In essence, decayed teeth can initiate significant medical complications. Radiotherapy and the removal of decayed and infected teeth can lead to postoperative dental complications of osteonecrosis, osteomyelitis and painful nonhealing tooth extraction sockets. The effect of radiation on oral mucosa leads to very painful prolonged episodes of oral mucositis. This is an extremely painful and debilitating condition with significant morbidity in the physiological and psychological dimensions. Another problem is the effect of radiation inducing 
dysplasia (basal cell carcinoma) on previously healthy facial tissues requiring further surgical intervention. The tissue also has a reduced blood supply so that subsequent grafting with local flaps is compromised. The radiation treatment may prolong the life expectancy of the individual but the enjoyment of living and eating food, reduced intimacy due to trigeminal neuropathic pain (and breast neuropathy) creates depression, anxiety, despair and hopelessness. A damaged face and breasts are seen by many patients as a damaged "self".

Translational research is urgently needed when considering the current unmet requirements for the prevention, improvement and reconstruction of tissue affected by radiation. Reconstruction of the face and breast with normal vasculature and nerve innervation would have a major beneficial impact on the medical and psychosocial health of patients. Combining the knowledge of stem cells and optimizing its cell growth medium to a therapeutic manufacturing scale for organoids clearly would be advantageous in enhancing positive patient outcomes after establishing safety and efficacy trials. Currently, orthopedic surgeons routinely remove infected and non-vital bone then later conduct bone regeneration using bone marrow augmentation that is dense with stem cells. Burns specialists use a similar approach of placing stem cell enriched skin grafts. Cancer surgeons of the breast and orofacial regions should be encouraged to perform regenerative procedures. The basis of regenerative medicine technology has shown efficacy and safety from these other medical specialists. The cost of the acquisition and delivery of stem cells has reduced markedly due to greater knowledge and implementation of clinically designed microfluidic separations and peptidic chemoattraction gels [55]. This allows the reconstructive surgeon to design and perform planned and careful incremental adjustments for facial and breast augmentation after ablative surgery and radiotherapy. Furthermore, the administration of stem cells is becoming simpler via injectable semi-viscous gels containing stem cells, peptides and polyphenols followed by crosslinking to achieve 3D gel stability, or direct 3D bioprinting. The peptides can help direct differentiation of stem cells to be specific to the donor tissue. The polyphenols can induce differentiation for maturing cells into functional tissue. Individual polyphenols from herbal medicines can be obtained by separation chromatography to yield pharmaceutically pure compounds for accurate dosage regimens either topically, oral systemic use, by inhalation or direct inclusion in biodegradable gels. The inhalational approach is particularly useful as the compound is absorbed rapidly and avoids pharmacokinetic first pass metabolism and elimination. Herbs however, may work better when all constituents of the plant are used as previously mentioned with green tea. In addition, the use of combination herbal formulas utilizing several plants for synergistic actions has been thoroughly tested in Traditional Chinese Medicine and European/British herbal medicine practice. The scope of research and practice using herbal formulas and the methods of application may dramatically improve outcomes after surgery and radiotherapy when considering the current extensive evidence base of knowledge of the polyphenols and phytotherapy. 
Table 1. Comparison of radiation dose of occupation and medical treatments. The radiation dose is measured in millisieverts (mSv) of effective dose [Jedick 2014].

\begin{tabular}{cc}
\hline Occupation/medical treatment & Radiation dose (mSv) \\
\hline 1 day of natural background radiation & $0.01 \mathrm{mSv}$ \\
Chest X-Ray & $0.1 \mathrm{mSv}$ \\
Mammogram & $0.7 \mathrm{mSv}$ \\
Pregnant aircrew maximum during pregnancy & $1 \mathrm{mSv}$ \\
Average human exposure per year & $3 \mathrm{mSv}$ \\
Aircrew per year & $6 \mathrm{mSv}$ \\
Body CT Scan & $10 \mathrm{mSv}$ \\
Radiation technician per year & $50 \mathrm{mSv}$ \\
Aircrew maximum exposure per year & $50 \mathrm{mSv}$ \\
Human maximum accumulated over a lifetime & $100 \mathrm{mSv}$ \\
Astronaut living on the International Space Station (6 month exposure) & $100 \mathrm{mSv}$ \\
Radiotherapy (head \& neck, breast) & $500 \mathrm{mSv}(50 \mathrm{~Gy})$ \\
Astronaut journey to Mars (6 month exposure) & $1000 \mathrm{mSv}$ \\
Acute Radiation Syndrome (fatal ARS exposure) & $1000-2000 \mathrm{mSv}$ \\
\hline
\end{tabular}

There are other applications of regenerative medicine for other forms of radiation (Table 1). The prevention of skin cancer receives major attention in Australia that has the highest skin cancer rate in the world. Solar radiation causes a high prevalence of melanoma and carcinoma in Australians of fair skinned Caucasian background. A topical cream composed of a proven and safe herbal compound mix would be widely used in the general population due to popular outdoor sports activities. Occupations involving outdoor activities such as building and construction, tourism, and pilots exposed to long duration flights may benefit. One particular use could be astronauts exposed to very high levels of cosmic radiation that would provide a greater level of protection. Cosmic radiation damage to human physiology has been identified by NASA astronauts as the greatest problem preventing space exploration. Successful space exploration is a prelude to commercial space mining and the acquisition of helium-3 from the moon for nuclear fusion technology and asteroid mining for example the Psyche 16 asteroid. An astronaut living on the International Space Station for six months is exposed to the same radiation level as a human's lifetime on earth [56].

\section{Conclusion}

In summary, there are currently techniques and compounds in regenerative medicine that clearly have the possibility to impact radiotherapy in several ways. Herbal polyphenols can radiosensitize cancer cells and yet be protective against its damaging effects to healthy tissues. Stem cells are novel and have intrinsic regenerative capability. Further identification of molecular agents to proliferate and differentiate tissue "on demand" has enormous potential to rectify the lack 
of treatments to date to counteract radiation induced injury.

\section{Conflicts of Interest}

The authors declare no conflicts of interest regarding the publication of this paper.

\section{References}

[1] Sung, H., Ferlay, J., Siegel, R.L., Laversanne, M., Soerjomataram, I., Jemal, A. and Bray, F. (2021) Global Cancer Statistics 2020: GLOBOCAN Estimates of Incidence and Mortality Worldwide for 36 Cancers in 185 Countries. CA: A Cancer Journal for Clinicians, 71, 209-249. https://doi.org/10.3322/caac.21660

[2] National Cancer Institute (n.d.) Survivorship. https://www.cancer.gov/publications/dictionaries/cancer-terms?cdrid=445089

[3] Ann, Y., Kim, H.H., Shin, H.J, Huh, M.O., Ahn, S.D. and Seo, B.K. (2009) Radiation-Induced Complications after Breast Cancer Radiation Therapy: A Pictorial Review of Multimodality Imaging Findings. Korean Journal of Radiology, 10, 496-507. https://doi.org/10.3348/kjr.2009.10.5.496

[4] Karlsen, J. (2019) Pneumonitis and Fibrosis after Breast Cancer Radiation. Abstracts Breast Cancer, Early Stage, 30, Article No. V74. https://doi.org/10.1093/annonc/mdz240.044

[5] Wei, J., Meng, L., Hou, X., Qu, C., Wang, B., Xin, Y. and Jiang, X. (2019) RadiationInduced Skin Reactions: Mechanism and Treatment. Cancer Management Research, 11, 167-177. https://doi.org/10.2147/CMAR.S188655

[6] Martin, H., Strong, E. and Spiro, R.H. (1970) Radiation-induced Skin Cancer of the Head and Neck. Cancer, 25, 61-71. https://doi.org/10.1002/1097-0142(197001)25:1\%3C61::AID-CNCR2820250110\%3E 3.0.CO;2-W

[7] Freites-Martinez. A., Shapiro. J., van den Hurk, C., Goldfarb, S., Jimenez, J.J., Rossi, A.M., et al. (2019) Hair Disorders in Cancer Survivors. Journal of the American Academy of Dermatology, 80, 1199-1213. https://doi.org/10.1016/j.jaad.2018.03.056

[8] Vickers, E.R. (2015) Neuropathic Orofacial Pain: Diagnosis and Multimodal Management. Medicine Today, 16, 16-24.

[9] Larsson, I.M., Sørensen, J.A. and Bille, C. (2017) The Post-mastectomy Pain Syndrome-A Systematic Review of the Treatment Modalities. The Breast Journal, 23, 338-343. https://doi.org/10.1111/tbj.12739

[10] Mills, E.P., Di Pietro, F., Alshelh, Z., Peck, C.C., Murray, G.M., Vickers, E.R. and Henderson, L.A. (2018) Brainstem Pain-Control Circuitry Connectivity in Chronic Neuropathic Pain. Journal of Neuroscience, 38, 465-473. https://doi.org/10.1523/JNEUROSCI.1647-17.2017

[11] Mills, E.P., Alshelh, Z., Kosanovic, D., Di Pietro, F., Vickers, E.R., Macey, P.M. and Henderson, L.A. (2020) Altered Brainstem Pain-Modulation Circuitry Connectivity during Spontaneous Pain Intensity Fluctuations. Journal of Pain Research, 13, 2223 2235. https://doi.org/10.2147/JPR.S252594

[12] Käsmann, L., Dietrich, A., Staab-Weijnitz, C.A., Manapov, F., Behr, J., Rimner, A., et al. (2020) Radiation-Induced Lung Toxicity-Cellular and Molecular Mechanisms of Pathogenesis, Management, and Literature Review. Radiation Oncology, 15, Article No. 214. https://doi.org/10.1186/s13014-020-01654-9

[13] Jones, R.E., Foster, D.S., Hu, M.S. and Longaker, M.T. (2019) Wound Healing and 
Fibrosis: Current Stem Cell Therapies. Transfusion, 59, 884-892. https://doi.org/10.1111/trf.14836

[14] Chen, Y., Liu, Y., Zhang, J., Liu, H., Wang, J., Liu, Q. and Zhang, Y. (2021) Three Dimensional Bioprinting Adipose Tissue and Mammary Organoids Feasible for Artificial Breast Structure Regeneration. Materials \& Design, 200, Article No. 109467. https://doi.org/10.1016/j.matdes.2021.109467

[15] Vickers, E.R., Karsten, E., Flood, J. and Lilischkis, R. (2014) A Preliminary Report on Stem Cell Therapy for Neuropathic Pain in Humans. Journal of Pain Research, 7, 255-263. https://doi.org/10.2147/JPR.S63361

[16] Egger, A., Tomic-Canic, M. and Tosti, A. (2020) Advances in Stem Cell-Based Therapy for Hair Loss. Cell R4-Repair, Replacement, Regeneration, and Reprogramming, 8, Article No. e2894.

[17] Vickers, E.R. (2017) An Analysis of the Safety and Efficacy of Topical Zinc-Thymulin to Treat Androgenetic Alopecia. Hair Therapy and Transplantation, 7, Article No. 147. https://doi.org/10.4172/2167-0951.1000147

[18] Wang, Q., Xie, Xi, S., Qian, F., Peng, X., Huang, J. and Tang, F. (2020) Radioprotective Effect of Flavonoids on Ionizing Radiation-Induced Brain Damage. Molecules, 25, Article No. 5719. https://doi.org/10.3390/molecules25235719

[19] Ghasemi, S., Xu, S., Nabavi, S.M., Amirkhani, M.A., Sureda, A., Tejada, S., et al. (2021) Epigenetic Targeting of Cancer Stem Cells by Polyphenols (Cancer Stem Cells Targeting). Phytotherapy Research, 35, 3649-3664. https://doi.org/10.1002/ptr.7059

[20] Gu, H.F., Mao, X.Y. and Du, M. (2020) Prevention of Breast Cancer by Dietary Polyphenols-Role of Cancer Stem Cells. Critical Reviews in Food Science and Nutrition, 60, 810-825. https://doi.org/10.1080/10408398.2018.1551778

[21] Younes, M., Aggett, P., Aguilar, F., Crebelli, R., Dusemund, B., Filipič, M., Frutos, M.J., et al. (2018) Scientific Opinion on the Safety of Green Tea Catechins. EFSA Journal, 16, Article No. e05239. https://doi.org/10.2903/j.efsa.2018.5239

[22] Yan, Y., Cook, J., McQuillan, J., Zhang, G., Hitzman, C.J., Wang, Y., Wiedmann, T.S. and You, M. (2007) Chemopreventive Effect of Aerosolized Polyphenon E on Lung Tumorigenesis in A/J Mice. Neoplasia, 9, 401-405. https://doi.org/10.1593/neo.07160

[23] Kang, Q., Zhang, X., Cao, N., Chen, C., Yi, J., Hao, L., Ji, Y., Liu, X. and Lu, J. (2019) EGCG Enhances Cancer Cells Sensitivity Under ${ }^{60} \mathrm{Co} \gamma$ Radiation Based on miR34a/Sirt1/p53. Food and Chemical Toxicology, 133, Article ID: 10807. https://doi.org/10.1016/j.fct.2019.110807

[24] Zhu, W., Xu, J., Ge, Y., Cao, H., Ge, X., Luo, J., Xue, J., Yang, H., Zhang, S. and Cao, J. (2014) Epigallocatechin-3-gallate (EGCG) Protects Skin Cells from Ionizing Radiation via Heme Oxygenase-1 (HO-1) Overexpression. Journal of Radiation Research, 55, 1056-1065. https://doi.org/10.1093/jrr/rru047

[25] Wang, Y., Li, M., Xu, X., Song, M., Tao, H. and Bai Y. (2012) Green Tea Epigallocatechin-3-Gallate (EGCG) Promotes Neural Progenitor Cell Proliferation and Sonic Hedgehog Pathway Activation During Adult Hippocampal Neurogenesis. Molecular Nutrition \& Food Research, 56, 1292-1303. https://doi.org/10.1002/mnfr.201200035

[26] Ud-Din, S., Wilgus, T.A., McGeorge, D.D. and Bayat, A. (2021) Pre-Emptive Priming of Human Skin Improves Cutaneous Scarring and is Superior to Immediate and Delayed Topical Anti-Scarring Treatment Post-Wounding: A Double-Blind Randomised Placebo-Controlled Clinical Trial. Pharmaceutics, 13, Article No. 510. https://doi.org/10.3390/pharmaceutics13040510 
[27] An, S.S., Kim, Y.O., Park, C.H., Lin, H. and Yoon, M.H. (2014) Antiallodynic Effect of Intrathecal Epigallocatechin-3-Gallate Due to Suppression of Reactive Oxygen Species Korean Journal of Anesthesiology, 67, 123-128. https://doi.org/10.4097/kjae.2014.67.2.123

[28] Jeon, J., Kim, J.H., Lee, C.K., Oh, C.H. and Song, H.J. (2014) The Antimicrobial Activity of (-)-Epigallocatehin-3-Gallate and Green Tea Extracts against Pseudomonas aeruginosa and Escherichia coli Isolated from Skin Wounds. Annals of Dermatolo$g y, 26,564-569$.

[29] Kale, A., Pişkin, Ö., Baş, Y., Aydın, B.G., Can, M., Elmas, Ö. and Büyükuysal, E.Ç. (2018) Neuroprotective Effects of Quercetin on Radiation-Induced Brain Injury in Rats. Journal of Radiation Research, 59, 404-410. https://doi.org/10.1093/jrr/rry032

[30] Ezzati, M., Yousefi, B., Velaei, K. and Safa, A. (2020) A Review on Anti-Cancer Properties of Quercetin in Breast Cancer. Life Sciences, 248, Article No. 117463. https://doi.org/10.1016/j.lfs.2020.117463

[31] Srivastava, S., Somasagara, R., Hegde, M., Nishana, M., Tadi, S.K., Srivastava, M., et al. (2016) Quercetin, a Natural Flavonoid Interacts with DNA, Arrests Cell Cycle and Causes Tumor Regression by Activating Mitochondrial Pathway of Apoptosis. Science Reports, 6, Article No. 24049. https://doi.org/10.1038/srep24049

[32] Li, X., Jin, Q., Yao, Q., Xu, B., Li, L., Zhang, S. and Tu, C. (2018) The Flavonoid Quercetin Ameliorates Liver Inflammation and Fibrosis by Regulating Hepatic Macrophages Activation and Polarization in Mice. Frontiers in Pharmacology, 9, Article No. 72. https://doi.org/10.3389/fphar.2018.00072

[33] Song, J-Y., Truong, D.V. and Yang, B. (2018) Quercetin Shows the Pharmacological Activity to Simultaneously Downregulate the Inflammatory and Fibrotic Responses to Tissue Injury in Association with its Ability to Target Multi-Kinases. Pharmacology, 102, 142-153. https://doi.org/10.1159/000490417

[34] Doersch, K.M. and Newell-Rogers, M.K. (2017) The Impact of Quercetin on Wound Healing Relates to Changes in Av and B1 Integrin Expression. Experimental Biology and Medicine, 242, 1424-1431. https://doi.org/10.1177\%2F1535370217712961

[35] Wang, Z., Zhang, G., Le, Y., Ju, J., Zhang, P., Wan, D., Zhao, Q., et al. (2020) Quercetin Promotes Human Epidermal Stem Cell Proliferation through the Estrogen receptor/ $\beta$-catenin/c-Myc/cyclin A2 Signaling Pathway. Acta Biochimica et Biophysica Sinica, 52, 1102-1110. https://doi.org/10.1093/abbs/gmaa091

[36] Chapman, H.A., Wei, Y., Montas, G., et al. (2020) Reversal of TGF $\beta 1$-Driven Profibrotic State in Patients with Pulmonary Fibrosis. New England Journal of Medicine, 382, 1068-1070. https://doi.org/10.1056/NEJMc1915189

[37] Ryan, J.L., Heckler, C.E., Ling, M., Katz, A., Williams, J.P., Pentland, A.P., et al. (2013) Curcumin for Radiation Dermatitis: A Randomized, Double-Blind, Placebo-Controlled Clinical Trial of Thirty Breast Cancer Patients. Radiation Research, 180, 34-43. https://doi.org/10.1667/RR3255.1

[38] Shabeeb, D., Musa, A.E., Abd Ali, H.S. and Najafi, M. (2020) Curcumin Protects against Radiotherapy-Induced Oxidative Injury to the Skin. Drug Design Development and Therapy, 14, 3159-3163. https://doi.org/10.2147/DDDT.S265228

[39] Saidi, A., Kasabova, M., Vanderlynden, L., Wartenberg, M., Kara-Ali, G.H., Marc, D., et al. (2019) Curcumin Inhibits the TGF- $\beta 1-D e p e n d e n t$ Differentiation of Lung Fibroblasts via Ppar $\gamma$-Driven Upregulation of Cathepsins B and L. Science Reports, 9, Article No. 491. https://doi.org/10.1038/s41598-018-36858-3

[40] Mansouri, K., Rasoulpoor, S., Daneshkhah, A., Abolfathi, S., Salari, N., Mohammadi, M., et al. (2020) Clinical Effects of Curcumin in Enhancing Cancer Therapy: A Sys- 
tematic Review. BMC Cancer, 20, Article No. 791. https://doi.org/10.1186/s12885-020-07256-8

[41] Xie, W., Kang, Z., Jiang, C. and Liu, N. (2019) Administration of Curcumin Alleviates Neuropathic Pain in a Rat Model of Brachial Plexus Avulsion. Pharmacology, 103, 324-331. https://doi.org/10.1159/000496928

[42] Sharifi, S., Vahed, S.Z., Ahmadian, E., Dizaj, S.M., Abedi, A., Khatibi, S.M.H., et al. (2019) Stem Cell Therapy: Curcumin Does the Trick. Phytotherapy Research, 33, 2927-2937. https://doi.org/10.1002/ptr.6482

[43] Zheng, J., Kulkarni, A., Wang, W., Garg, S., Prather, P.L. and Hauer-Jensen, M. (2014) Palmitoylethanolamide Regulates Development of Intestinal Radiation Injury in a Mast Cell-Dependent Manner. Digestive Diseases and Sciences, 59, 2693 2703. https://doi.org/10.1007/s10620-014-3212-5

[44] Sarnelli, G., Gigli, S., Capoccia, E., Iuvone, T., Cirillo, C., Seguella, L., Nobile, N., et al. (2016) Palmitoylethanolamide Exerts Antiproliferative Effect and Downregulates VEGF Signaling in Caco-2 Human Colon Carcinoma Cell Line Through a Selective PPAR-a-Dependent Inhibition of Akt/mTOR Pathway. Phytotherapy Research, 30, 963-970. https://doi.org/10.1002/ptr.5601

[45] De Gregorio, D., Manchia, M., Carpiniello, B., Valtorta, F., Nobile, Gobbi, G. and Comai, S. (2019) Role of Palmitoylethanolamide (PEA) in Depression: Translational Evidence. Specials Section "Translational and Neuroscience Studies in Affective Disorders". Journal of Affective Disorders, 255, 195-200. https://doi.org/10.1016/j.jad.2018.10.117

[46] Masatsugu, O., Shunsuke, O., Hidetaka, H., Koji, Y., Qingjie, F., Osamu, M., Goki, S., et al. (2018) Palmitoylethanolamide Ameliorates Carbon Tetrachloride-Induced Liver Fibrosis in Rats. Frontiers in Pharmacology, 9, Article No. 709. https://doi.org/10.3389/fphar.2018.00709

[47] Pagano, E., Venneri, T., Lucariello, G., Cicia, D., Brancaleone, V., Nanì, M., Cacciola, F., et al. (2021) Palmitoylethanolamide Reduces Colon Cancer Cell Proliferation and Migration, Influences Tumor Cell Cycle and Exerts in Vivo Chemopreventive Effects. Cancers, 13, Article No.1923. https://doi.org/10.3390/cancers13081923

[48] Vickers, E.R., Cousins, M., Walker, S. and Chisholm, K. (1998) Analysis of $50 \mathrm{~Pa}-$ tients with Atypical Odontalgia: A Preliminary Report on Pharmacological Procedures for Diagnosis and Treatment. Oral Surgery, Oral Medicine, Oral Pathology, Oral Radiology and Endodontics, 85, 24-32. https://doi.org/10.1016/S1079-2104(98)90393-6

[49] Zhang, S., Wang, D., Huang, J., Hu, Y. and Xu, Y. (2020) Application of Capsaicin as a Potential New Therapeutic Drug in Human Cancers. Journal of Clinical Pharmacy and Therapeutics, 45, 16-28. https://doi.org/10.1111/jcpt.13039

[50] Gangabhagirathi, R. and Joshi, R. (2015) Antioxidant Activity of Capsaicin on Radiation-Induced Oxidation of Murine Hepatic Mitochondrial Membrane Preparation. Research and Reports in Biochemistry, 5, 163-171.

https://doi.org/10.2147/RRBC.S84270

[51] Mendivil, E.J., Sandoval-Rodriguez, A., Zuñiga-Ramos, L.M., Santos-Garcia, A. and Armendariz-Borunda J. (2019) Capsaicin and Sulforaphane Prevent Experimental Liver Fibrosis via Upregulation of Peroxisome Proliferator-Activated Receptor Gamma and Nuclear Factor (Erythroid-Derived 2)-Like 2. Journal of Functional Foods, 52, 382-388. https://doi.org/10.1016/j.jff.2018.11.014

[52] Wallengren, J., Chen, D. and Sundler, F. (1999) Neuropeptide-Containing C-Fibres and Wound Healing in Rat Skin. Neither Capsaicin nor Peripheral Neurotomy Af- 
fect the Rate of Healing. British Journal of Dermatology, 140, 400-408. https://doi.org/10.1046/j.1365-2133.1999.02699.x

[53] Yuan, M., Zhao, L., Li, Y., Gao, X., Zhang, B., Zhang, D. and Li, Y. (2021) Capsaicin on Stem Cell Proliferation and Fate Determinations-A Novel Perspective. Pharmacological Research, 167, Article ID: 105566.

https://doi.org/10.1016/j.phrs.2021.105566

[54] Marazziti, D., Baroni, S., Catena-Dell'Osso, M., Schiavi, E., Ceresoli, D., Conversano, C., Dell'Osso, L. and Picano, E. (2012) Cognitive, Psychological and Psychiatric Effects of Ionizing Radiation Exposure. Current Medicinal Chemistry, 19, 1864-1869. https://doi.org/10.2174/092986712800099776

[55] Vickers, E.R. (2020) Peptidic Alginate-Based Hydrogels Demonstrate Chemotaxis and Expansion to Adipose Tissue Derived and Blood Derived Stem Cells. Journal of Biomaterials and Nanobiotechnology, 11, 245-259.

https://doi.org/10.4236/jbnb.2020.114016

[56] Jedick, R. (2014) Cosmic Radiation. https://goflightmedicine.com/cosmic-radiation/ 